\title{
Study of the solution for disinfection by supplying residual chlorine in the distribution networks of drinking water
}

\author{
Dinh Xuan Vinh Phan ${ }^{1,}$, Anh Tuan Vo ${ }^{1}$, Huynh Thuy Ngan Nguyen ${ }^{1}$, Thi My Hanh Ho ${ }^{1}$ \\ ${ }^{1}$ University of Architecture Ho Chi Minh City, 196 Pasteur street, ward 6, District 3, Ho Chi Minh \\ City, Vietnam
}

\begin{abstract}
This paper proposes a solution to ensure quality of drinking water in water supply network by increasing chlorine concentration, reaching concentration level to disinfect water according to Ministry of Health regulation for water supply. Specifically, this study has been carried out at Tan Kien water supply station, Binh Chanh district, Ho Chi Minh City, by installing an additional chlorine station along the pipeline. As a result, after installation, the residual chlorine concentration has reached required concentration and has maintained the stability of its level. The chlorine station is an integrated, compact solution suitable for similar water supply areas: far from water supply plants, inadequate chlorine concentration and limited space for installation of chlorine system.
\end{abstract}

\section{Introduction}

Ho Chi Minh City transmission and distribution water network includes 06 water supply areas. It is characterized by urban water supply areas located far from the water treatment plants, clean water distribution and distribution networks that are not planned and are degraded due to relatively high pipeline life and asynchronous pipeline materials, high water leakage rate, these factors directly affect to drinking water quality. Tan Kien water supply area, Binh Chanh district is supplied with water from Tan Hiep water plant that $30 \mathrm{~km}$ away, quality of water is reflected by people live there are often not good, high color and high turbidity, and sampling surveys show frequent concentrations of chlorine in the range 0.0 $0.2 \mathrm{mg} / \mathrm{l}$.

Meanwhile, according to the regulation of Vietnamese standard QCVN 02: 2009 of the Ministry of Health on drinking water quality and Sai Gon Water Supply Corporation (SAWACO) the residual chlorine concentration in drinking water is $0.3-0.5 \mathrm{mg} / 1$.In storage tank and distribution network, drinking water must be kept safe from microbial contamination. Frequently, slippery films of bacteria, known as biofilms, develop on the inside walls of pipes and storage containers. Among disinfection techniques, chlorination is unique in that a pre-determined chlorine concentration may be designed to remain in treated water as a measure of protection against harmful microbes encountered after leaving the treatment facility [1-5].

\footnotetext{
*Corresponding author: xuanvinh09@gmail.com
} 
So problem is chlorine concentrate level in this water supply area to meet the SAWACO requirements. Due to water supply area is far from the plant, it is not feasible to raise chlorine concentrate at water plant to meet whole water supply network chlorine concentrate requirement, as the chlorine concentration of water in the area near the plant is very high. The author proposes additional chlorine solution at the Tan Kien water supply area. The study has conducted a survey on the current state of the water supply network, sampling chlorine concentrations to determine the location of the chlorine as well as the water flow meter. Chlorine in granular form supplementation station has been set up at a fixed concentration, the amount of chlorine has fluctuated according to the flow rate into the water supply area for efficient and economical chlorination.

Chlorinationis a popular waterdisinfectant method. However, effluent chlorination results in the formation of mutagenic/carcinogenic disinfection by-products (DBPs) deriving from the reaction of the chlorine with organic compounds in wastewater [16]. Therefore, dechlorination followed by chlorination should be done, or alternative safe disinfectant should be used [2], in this study that use disinfection chemical is ChloRun not create the byproducts, which is a complete for drinking water.

\section{Materials and methods}

\subsection{Status of Tan Kien water supply area}

The Tan Kien water supply area is studied on Duong Dinh Cuc street, includes two HDPE OD (outside diameter) $160 \mathrm{~mm}$ and OD $125 \mathrm{~mm}$ water supply pipes along two site of this street, water source form transmission pipeline on National Highway 1A. With hydraulic parameters as follows:

- Quantity of customer connections: 860 connections

- $\quad$ Maximum pipeline length: 1700 meters

- Average flow: $1200 \mathrm{~m} 3 /$ day

- Water pressure at the beginning of the network: $0.3 \div 1.5$ bars

Water sampler was taken to check the residual chlorine concentration in water, water sample taken after the water meter, at starting and end point of the network, measured by colorimetric method. Average residual chlorine of sample on network is as follows:

- Inlet: $0.0 \div 0.2 \mathrm{mg} / \mathrm{L}$ (Most of the time there is no residual chlorine in the water)

- $\quad$ End of the network: $0.0 \div 0.1 \mathrm{mg} / \mathrm{L}$

The sampling results indicate need to add chlorine at the start of the network to raise reach 0.3 to $0.5 \mathrm{mg} / \mathrm{l}$ chlorine concentrate as SAWACO requirement. The author proposes to install an additional chlorine station, proper location for installing chlorine is the land of public works. Because Duong Dinh Cuc street is mainly residential and commercial, the author chooses the installation location at the old Tan Kien water supply station 2, about 100m from the north of Duong Dinh Cuc street.

The Tan Kien water supply station 2 used to treat underground water to serve local people, but later, according to the policy of SAWACO, not using underground water but using water directly from the city water supply network, it was stopped. This location is well suited for the installation of a chlorination station.

Chlorine additional position is on OD $160 \mathrm{~mm}$ water supply, after this position pipeline is divided into two branches to serve Tan Kien water supply area. 


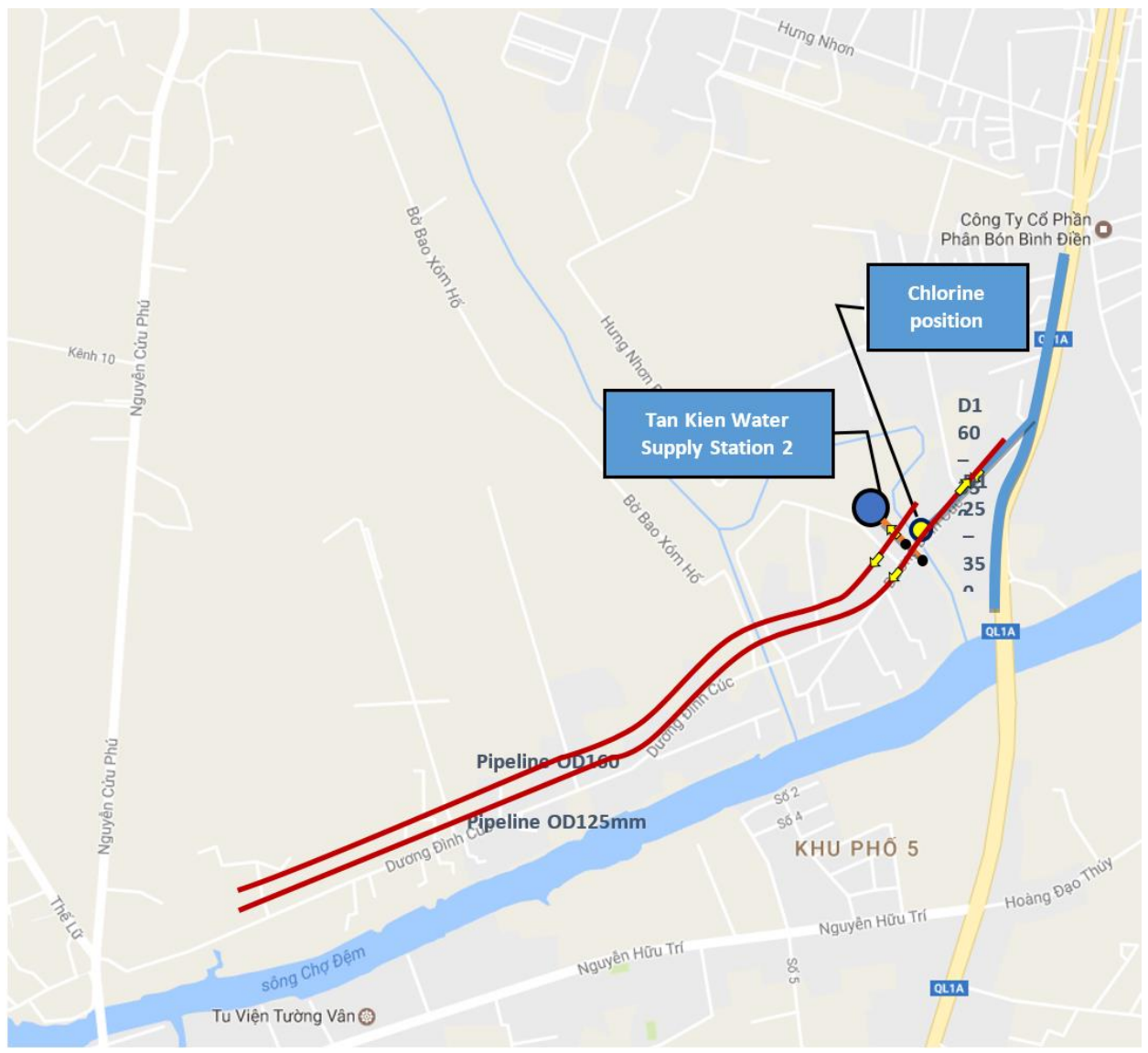

Fig. 1. Location of Tan Kien water supply area.

\subsection{Basis for selection device and Chlorine Granules (ChloRun)}

\subsubsection{Chlorine Granules (ChloRun)}

Most popular chlorine system at water treatment plants in Vietnam are using elemental chlorine (liquid chlorine), Chlorine gas can be pressurized and cooled to change it into a liquid so that it can be shipped and stored. When liquid chlorine is released, it quickly turns into a gas that uses for water disinfection. Advantages of elemental chlorine are traditional system so chlorine system can be inherited existing infrastructure equipment, low price and high concentration -above $99 \%$ chlorine. However, the biggest disadvantage is risk of chlorine gas leakage, chlorine gas at high concentrations is extremely dangerous and poisonous for all living organisms [19]. At factories to ensure safety when using elemental chlorine, piping and valves often require very strict sealing, and leak detection systems, as well as chlorine gas neutralization system that are relatively expensive. Other commonly chemical disinfection areCalcium Chloride (56\% Chlorine), Liquid bleach (10$12 \%$ Chlorine).

A typical chlorinator system use elemental chlorine: 


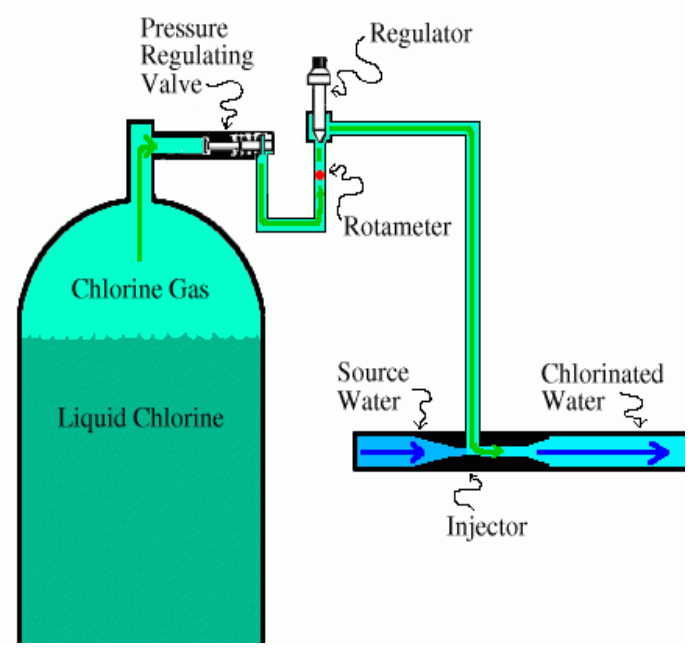

Fig. 2. Typical chlorinator system.

In this study, due to need for an integrated and safe chlorination systemlocated close to the residential area, there is no need for substantial investment in infrastructure, the author recommends using Chlorun form Brand ICL-Israel.

This is chlorine in granularform of $56 \%$ concentration, with the advantage of being able to be stored at normal conditions in container, thus eliminating the risk of leakage; Full water solubility without the need for additional stirrers, no by-products and no change in $\mathrm{pH}$ (when used in low concentrations). Chemical formula ofChloRun:Hypochlorite acid $\mathrm{HOCl}$ (Disinfectation AGENT)+ (Iso) cyanurate-NaCl2(NCO) $3 * 2 \mathrm{H} 2 \mathrm{O}$.

$$
\begin{aligned}
& \mathrm{NaCl} 2(\mathrm{NCO}) 3 * \mathrm{H} 2 \mathrm{O}+\mathrm{H} 2 \mathrm{O} \longrightarrow 2 \mathrm{HOCl}+\mathrm{Na}++\mathrm{C} 3 \mathrm{~N} 3 \mathrm{O} 3 \mathrm{H} 3 \\
& \text { Chlorun water Hypochlorous Cyanuric } \\
& \text { Acid acid } \\
& \text { (Killing agent) }
\end{aligned}
$$

Hypochlorous acid $(\mathrm{HOCl})$ is reactive and oxidizes a variety of organic and inorganic materials in the water.

$\mathrm{HOCl}$ is reduced to hydrochloric acid $(\mathrm{HCl})$ and hypochlorite ion $(\mathrm{OCl}-)$.

$$
\mathrm{HOCl}<-->\mathrm{HCl}+\mathrm{OCL}^{-}
$$

Hypochlorous acid ( $\mathrm{HOCl})$ is considerably more active as micro biocide than hypochlorite ion (OCl-).

The hypochlorite ion has about one hundredth of the biological effect of $\mathrm{HOCl}$; therefore greater bacterial control occurs in acid solutions than alkaline solutions at the same chlorine levels. The PRICLEAR is excellent micro biocide for neutral and slightly alkaline water.

$\mathrm{HOCl}$ has a similar chemical structure of water $(\mathrm{HOH})$ and electrically natural. These factors enable the compound to penetrate through the cell wall similar way to water.

On the other hand, OCl- has a very different structure and is electrically charged, making it difficult to diffuse to the cell wall. 
Table 1. Comparing different chlorine products.

\begin{tabular}{|l|c|c|c|c|}
\hline & ChloRun & $\begin{array}{c}\text { Calcium } \\
\text { Chloride }\end{array}$ & Liquid bleach & $\begin{array}{c}\text { Elemental } \\
\text { chlorine }\end{array}$ \\
\hline Concentration & $56 \%$ & $65 \%$ & $10-12 \%$ & $100 \%$ \\
\hline High chlorine content & $\times$ & & & $\times$ \\
\hline Easy to use & $\times$ & $\times$ & $\times$ & $\times$ \\
\hline No cloudy water & $\times$ & & $\times$ & $\times$ \\
\hline No scaling & $\times$ & & & \\
\hline Long storage life & $\times$ & $\times$ & & \\
\hline No messy spills & $\times$ & $\times$ & & \\
\hline $\begin{array}{l}\text { Safe to store and } \\
\text { handle }\end{array}$ & $\times$ & $\times$ & & \\
\hline $\begin{array}{l}\text { Less total dissolves } \\
\text { solids }\end{array}$ & & & & \\
\hline
\end{tabular}

\subsubsection{Chlorun system}

To mix and inject chlorine intopipeline network at required concentration, the author recommends using the chlorinator system is Chlorun system fromICL - Israel. The Chlorun system machine is very integrated, simple operation, the principle is Chlorine Granules solid in hopper container, under vibration will fall into the mixing tank (the concentration in the stirring tank can be set in range 1-10\% chlorine solution under hydraulic motion, and then a dosing pump draws chlorine solution and enters the pipeline with amount fluctuate depend on pipeline flow rate. The Chlorun system know that flow rate thank for recorded from water meter, continuous flow signal form water meter to the Chlorun system to regulate the dosing pump flow.

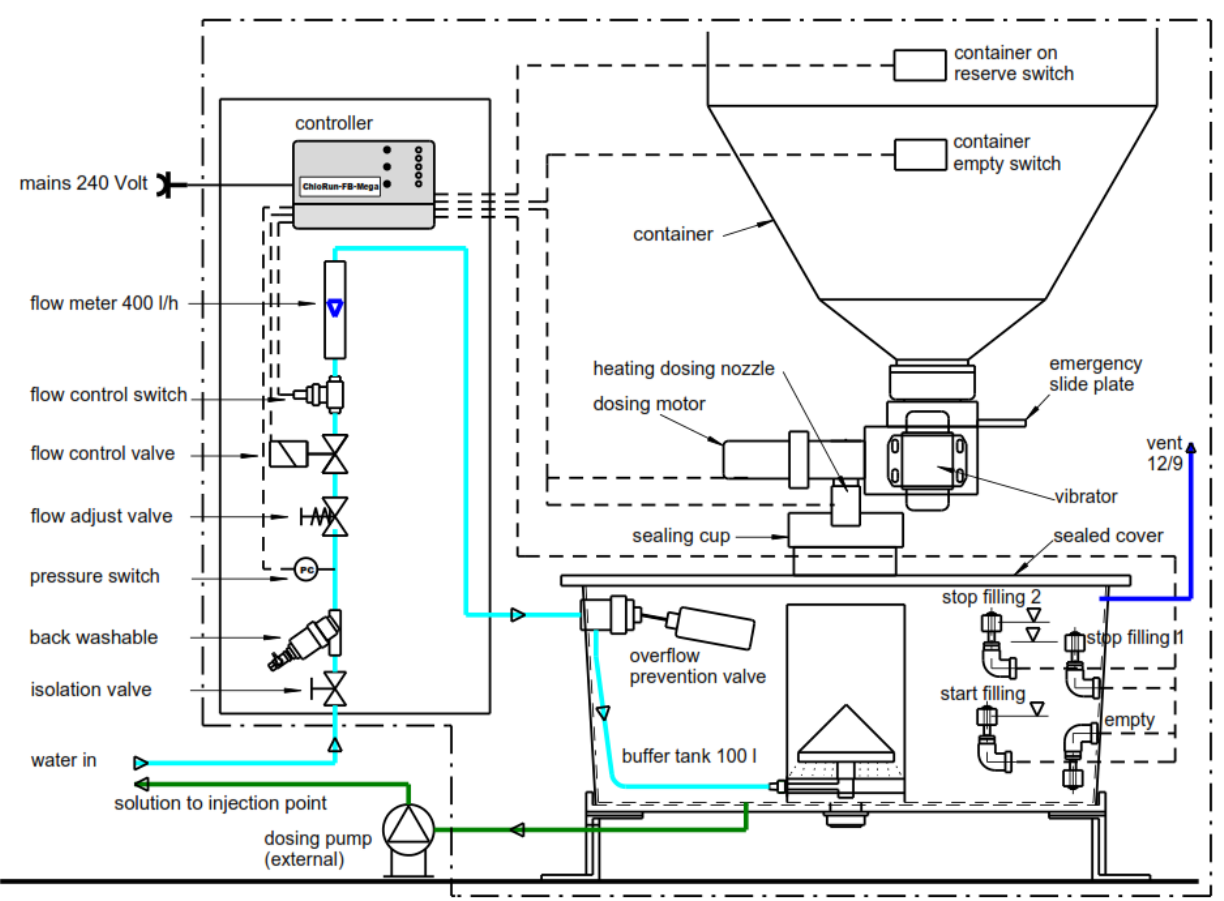

Fig. 3. Diagram of Chlorinator structure -ICL. 


\subsection{Model setting}

Input parameters

- Average flow rate: $800 \mathrm{~m} 3 /$ day $(33.3 \mathrm{~m} 3 / \mathrm{h})$

- Water pressure: $0.3 \div 1.5$ bars

- Existing chlorine levels: $0.0 \div 0.2 \mathrm{mg} / \mathrm{L}$

- Chlorine concentration requirement:

+ Inlet position: $0.3 \div 0.5 \mathrm{mg} / \mathrm{L}$

+ Critical point: $\geq 0.3 \mathrm{mg} / \mathrm{L}$

- Diameter of pipeline at chlorine adding position: OD160 HDPE (no flow meter at this location)

- Installation location of Chlorine Mixing System: Tan Kien Water Supply Station 2 (125m from the north of Duong Dinh Cuc street).

- Chlorine adding position on Duong Dinh Cuc street.

Technical solution: application of granular chlorine system.

The ChloRun system automatically dissolves Chlorine granules, prepares solution and add into water supply system with the flow regulated based on real usage.

Calculation in the most unfavorable case - the residual chlorine concentration is $0 \mathrm{mg} / \mathrm{L}$, to reach the residual chlorine required, author proposes add $0.5 \mathrm{mg} / 1$ chlorine solution concentration (this data may vary according to actual conditions)

Operating principle.

- Using booster pump from the internal Tan Kien water supply station 2 to increase the input pressure for the mixer, ensuring input water pressure $>1.5$ bar.

- Chlorine Dissolve Controller is installed to control the $1.5 \%$ chlorine solution $(15,000.0$ $\mathrm{mg} / \mathrm{l})$ in mixing tank.

- A DN150mm Electromagnetic flow meter is installed on the piping to measure the flow and send back flow pulse signal to the Chlorun system.

- The Chlorun system receives the flow signal from the water meter and controls the dosing pump to inject $1.5 \%$ chlorine solution into the pipeline continuously depending on the flow rate, ensuring the stability of $0.5 \mathrm{mg} / 1$ chlorine concentration.

- A dosing pump placed pump chlorine solution $1.5 \%$ through a $125 \mathrm{~m}$ long pipe to OD160mm water supply pipeline.

- The regulator will send a warning when ChloRun container hopper is empty or when there are system fails.

ChloRun power system calculation:

ChloRun solidin container hopper: maximum $35 \mathrm{~kg}$

Concentration of solution after mixing: $1.5 \%=15,000 \mathrm{mg} / \mathrm{L}$

Residual chlorine concentration requirement: $0.5 \mathrm{mg} / \mathrm{L}$

The amount of ChloRun solution (1.5\%) required at average flow: $33,300(\mathrm{~L} / \mathrm{h}) * 0.5$ / $15,000 /=1.1(\mathrm{~L} / \mathrm{h})$ or $(\mathrm{kg} / \mathrm{h})$.

The amount of ChloRun solution (1.5\%) required during the day: $800,000(\mathrm{~L}) * 0.5 / 15,000$ $=26.7 \mathrm{~L}(\mathrm{~kg})$

The amount of solution are injected into the pipeline and regulated by flow rate through the water meter, via the flow signal from the electromagnetic meter.

The amount of ChloRun particles consumed every day: $26.7 \mathrm{~kg} * 1.5 \% / 56 \%=0.72 \mathrm{~kg}$ 


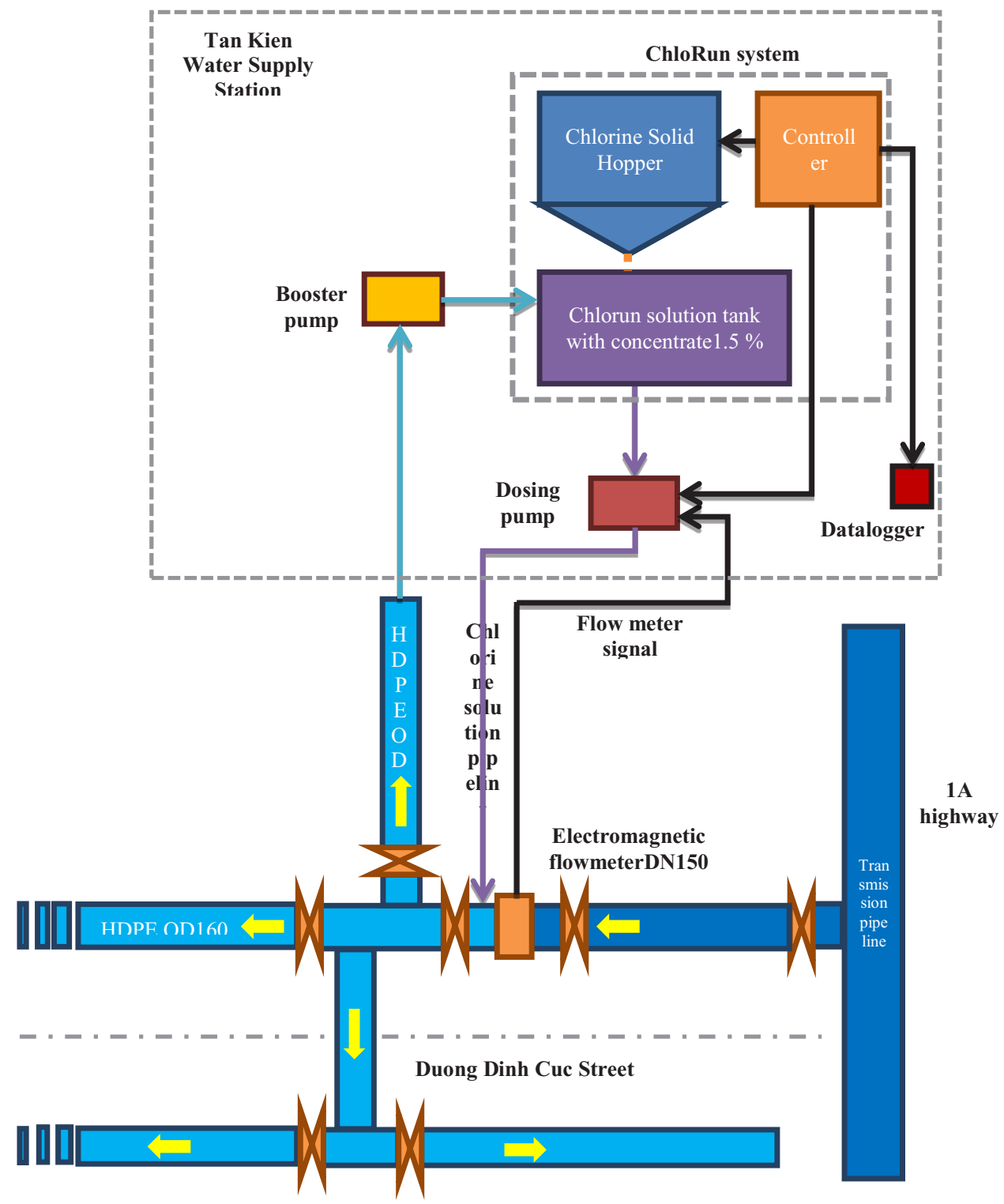

Fig. 4. Diagram of Chlorinating system at Tan Kien.

\section{Results}

After system operating, to ensure that chlorine concentrate under control, the author have set a change in chlorine concentrate level respectively at $0.2 \mathrm{mg} / 1,0.4 \mathrm{mg} / 1,0.5 \mathrm{mg} / 1$ and $0.6 \mathrm{mg} / 1$ to monitor change of residual chlorine concentration in water.

The flow of the chlorine dosing pump varies with the flow at the water meter as follows: 

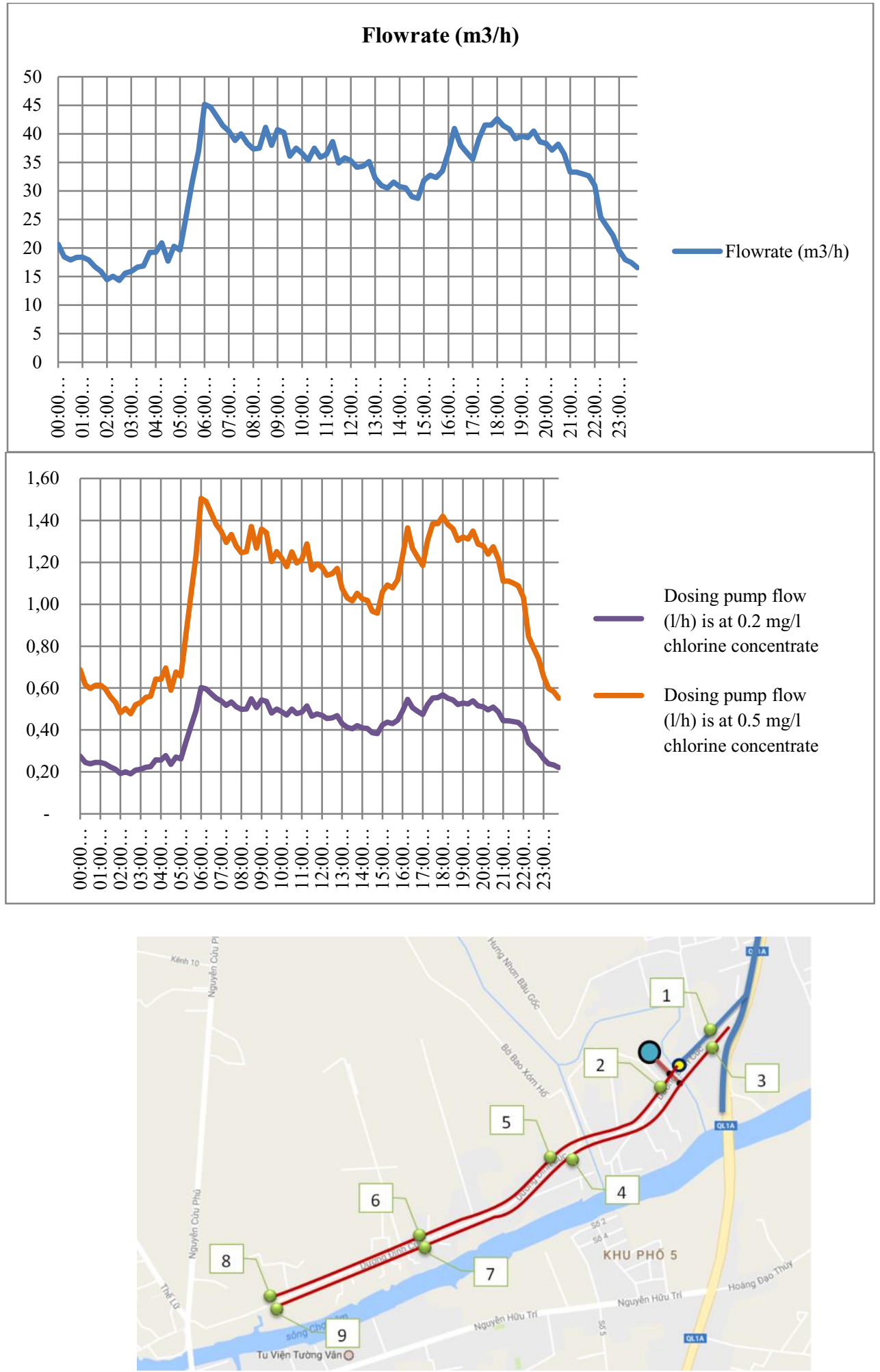

Fig. 5. Diagram of water sampling positions. 
Table 2. Chlorine sampling and $\mathrm{pH}$ levels results after 2 months.

\begin{tabular}{|c|c|c|c|c|c|c|c|c|c|c|c|c|c|c|c|c|c|c|c|c|c|}
\hline \multirow{3}{*}{$\begin{array}{l}\mathrm{N} \\
\mathrm{o}\end{array}$} & \multirow{3}{*}{ 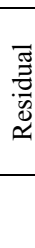 } & \multirow{3}{*}{$\stackrel{0}{\pi}$} & \multirow{3}{*}{$\stackrel{\Xi}{\Xi}$} & \multicolumn{18}{|c|}{$\begin{array}{l}\text { Chlorine residual and pHsample point } \\
(\mathrm{mg} / \mathrm{L}) / \mathrm{pH}\end{array}$} \\
\hline & & & & \multicolumn{2}{|c|}{1} & \multicolumn{2}{|c|}{2} & \multicolumn{2}{|c|}{3} & \multicolumn{2}{|c|}{4} & \multicolumn{2}{|c|}{5} & \multicolumn{2}{|c|}{6} & \multicolumn{2}{|c|}{7} & \multicolumn{2}{|c|}{8} & \multicolumn{2}{|c|}{9} \\
\hline & & & & $\bar{v}$ & $\stackrel{T}{2}$ & $\vec{U}$ & $\stackrel{\mathbb{I}}{\mathrm{z}}$ & $\bar{U}$ & $\vec{I}$ & $\bar{U}$ & $\stackrel{\pi}{2}$ & $\bar{U}$ & 焉 & $\bar{U}$ & 胥 & $\bar{U}$ & $\frac{\pi}{2}$ & $\bar{u}$ & 焉 & $\widetilde{U}$ & $\stackrel{\pi}{2}$ \\
\hline- & 0 & 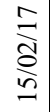 & ถี & $\stackrel{8}{0}$ & $\stackrel{n}{n}$ & $\stackrel{0}{0}$ & $\stackrel{n}{n}$ & $\stackrel{\sigma}{\circ}$ & $\underset{\sim}{\sim}$ & & & & & & & & & & & & \\
\hline$N$ & 0 & 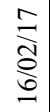 & ळ & $\stackrel{0}{0}$ & $\stackrel{n}{n}$ & $\stackrel{0}{\circ}$ & $\stackrel{n}{r}$ & $\hat{\circ}$ & $\stackrel{n}{n}$ & & & & & & & & & & & & \\
\hline$m$ & 0 & 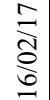 & 守 & $\stackrel{0}{0}$ & $\stackrel{\leftrightarrow}{n}$ & $\stackrel{n}{\circ}$ & $\stackrel{\sim}{n}$ & ठ̊. & $\underset{\sigma}{\sim}$ & & & & & & & & & & & & \\
\hline$\nabla$ & ชี & 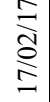 & ळ̊ & $\stackrel{n}{0} \cdot$ & $\stackrel{8}{i}$ & $\frac{2}{0}$ & $i n$ & $\stackrel{ \pm}{0}$ & $\stackrel{n}{n}$ & $=$ & $\stackrel{n}{n}$ & $\stackrel{2}{2}$ & $\stackrel{n}{n}$ & $\stackrel{\delta}{0}$ & $\stackrel{n}{n}$ & 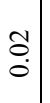 & $\stackrel{n}{n}$ & $\stackrel{\circ}{\circ}$ & $\hat{n}$ & $\stackrel{\Xi}{\circ}$ & $\stackrel{n}{n}$ \\
\hline in & ָั & 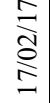 & 寻 & $\stackrel{0}{\circ}$ & $\stackrel{n}{n}$ & $\tilde{\sigma}$ & $\stackrel{n}{n}$ & $\stackrel{0}{\circ}$ & $\stackrel{n}{n}$ & $=$ & in & $\stackrel{m}{0}$ & $\stackrel{n}{r}$ & $\stackrel{\overbrace{}}{0}$ & in & o̊. & $i n$ & $\stackrel{\leftrightarrow}{\circ}$ & $\stackrel{n}{r}$ & $\tilde{O}$ & in \\
\hline$r$ & ֻै & 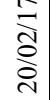 & 寻 & $\stackrel{0}{0}$ & $\stackrel{n}{n}$ & กิ & 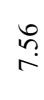 & $\frac{n}{0}$ & $\begin{array}{l}\vec{\sigma} \\
\sim\end{array}$ & $\stackrel{\circ}{\circ}$ & $\stackrel{\sigma}{r}$ & $\ddot{\circ}$ & $\underset{\sim}{\sigma}$ & $\stackrel{\circ}{\circ}$ & $\stackrel{\vec{\sigma}}{\sim}$ & $\ddot{\circ}$ & $\underset{\sim}{\sigma}$ & $\stackrel{\sigma}{\circ}$ & $\stackrel{\vec{\sim}}{\sim}$ & $\ddot{\circ}$ & $\underset{\sim}{\stackrel{\sigma}{r}}$ \\
\hline$\infty$ & $\stackrel{t}{0}$ & $\begin{array}{l}\underset{\delta}{\delta} \\
\underset{\sim}{\sim}\end{array}$ & हี & $\stackrel{0}{0}$ & in & $\stackrel{\text { I }}{0}$ & $\underset{\sigma}{\stackrel{\sigma}{r}}$ & 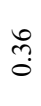 & $\underset{r}{\sigma}$ & $\stackrel{?}{0}$ & $\stackrel{\overbrace{}}{\sim}$ & m. & $\sqrt[n]{n}$ & $\vec{m}$ & 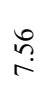 & ఝి & $\ddot{n}$ & $\stackrel{\text { J }}{0}$ & $\stackrel{t}{n}$ & 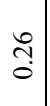 & 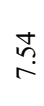 \\
\hline$\sigma$ & $\stackrel{t}{0}$ & 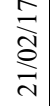 & ถี & $\stackrel{2}{2}$ & $\underset{\sigma}{\sim}$ & $\stackrel{?}{+}$ & 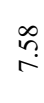 & $\stackrel{n}{?}$ & $\underset{r}{\vec{r}}$ & $\stackrel{?}{3}$ & $\stackrel{i}{r}$ & $\bar{m}$ & $\stackrel{t}{n}$ & సે & in & 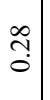 & $\stackrel{n}{n}$ & ઼ָ & $\stackrel{\leftrightarrow}{r}$ & $\vec{\jmath}$ & $\stackrel{\infty}{n}$ \\
\hline 으 & $\stackrel{t}{0}$ & 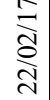 & 동 & $\begin{array}{l}0 \\
0 \\
0\end{array}$ & $i n$ & : & 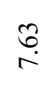 & $\stackrel{+}{3}$ & $\stackrel{n}{n}$ & กี & $\stackrel{n}{n}$ & $\stackrel{m}{?}$ & $\stackrel{\hat{\sigma}}{r}$ & ?ु & $\stackrel{\vec{b}}{\stackrel{r}{0}}$ & $\tilde{3}$ & $n$ & $\begin{array}{l}0 \\
\text { กิ }\end{array}$ & $\stackrel{n}{n}$ & 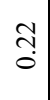 & $\stackrel{n}{2}$ \\
\hline$=$ & $\stackrel{t}{0}$ & 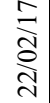 & 寻 & $\begin{array}{l}8 \\
0\end{array}$ & $\stackrel{8}{:}$ & $\vec{\nabla}$ & $\stackrel{n}{n}$ & ?ै? & $\begin{array}{l}8 \\
i \\
\therefore\end{array}$ & $\stackrel{+}{?}$ & $\stackrel{\vec{\sim}}{\sim}$ & $\bar{m}$ & 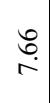 & 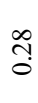 & ț & ते & $\stackrel{\circ}{:}$ & 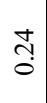 & $\stackrel{3}{r}$ & 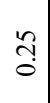 & $\stackrel{\vec{\sim}}{\sim}$ \\
\hline$\simeq$ & $\stackrel{\circ}{\circ}$ & 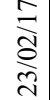 & 离 & $\stackrel{0}{0}$ & $\stackrel{\sim}{n}$ & $\stackrel{\sigma}{0}$ & $\stackrel{\bullet}{\sim}$ & ñ & $\underset{\sim}{\sigma}$ & ñ & $\stackrel{\overbrace{}}{r}$ & ก̂? & 莒 & in & $\stackrel{n}{n}$ & กิ & $\stackrel{n}{n}$ & $\underset{+}{+}$ & $\stackrel{\infty}{\stackrel{n}{r}}$ & f. & $\stackrel{6}{\circ}$ \\
\hline
\end{tabular}




\begin{tabular}{|c|c|c|c|c|c|c|c|c|c|c|c|c|c|c|c|c|c|c|c|c|c|}
\hline$\cong$ & $\stackrel{0}{\circ}$ & $\frac{\sqrt{2}}{\stackrel{\curvearrowright}{\curvearrowright}}$ & 드 & $\stackrel{\circ}{\circ}$ & $\sqrt[n]{r}$ & $\stackrel{8}{8}$ & $\underset{r}{\stackrel{J}{r}}$ & 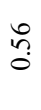 & $\underset{r}{6}$ & กี & $\stackrel{\sim}{r}$ & $\bar{n}$ & in & $\stackrel{\infty}{+}$ & $\tilde{n}$ & $\stackrel{0}{0}$ & $\underset{r}{\hat{r}}$ & $\stackrel{\leftrightarrow}{+}$ & $\underset{r}{\sigma}$ & $\stackrel{\infty}{\dddot{n}}$ & $\stackrel{+}{r}$ \\
\hline \pm & $\stackrel{+}{\circ}$ & 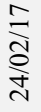 & ஜే & $\begin{array}{l}\infty \\
\stackrel{0}{0} \\
0\end{array}$ & $\underset{\sim}{\stackrel{\circ}{r}}$ & $\stackrel{\text { f }}{\circ}$ & $\stackrel{n}{n}$ & 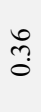 & ก๊ & m. & $\underset{r}{n}$ & $\stackrel{?}{0}$ & $\underset{\sim}{\sigma}$ & ঙ্ & $\stackrel{\circ}{\sim}$ & $\stackrel{n}{0}$ & $\stackrel{\infty}{\mathfrak{n}}$ & $\stackrel{\overbrace{}}{0}$ & in & $\stackrel{+ָ}{\stackrel{+}{0}}$ & $\begin{array}{l}n \\
r\end{array}$ \\
\hline$\cong$ & $\stackrel{+}{0}$ & 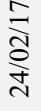 & 寻 & $\begin{array}{l}\circ \\
\stackrel{\circ}{\circ}\end{array}$ & $\stackrel{\sim}{r}$ & $\stackrel{+}{+}$ & $\hat{r}$ & $\stackrel{n}{n}$ & ñ & ?ֶ? & $\stackrel{\infty}{n}$ & $\stackrel{m}{0}$ & $\underset{\sim}{\sigma}$ & $\stackrel{n}{0}$ & $\stackrel{\infty}{\mathfrak{n}}$ & ?̊? & $\begin{array}{l}\stackrel{n}{r} \\
\curvearrowleft\end{array}$ & 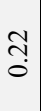 & $\underset{r}{\bar{\sigma}}$ & $\stackrel{\text { ָ̊ }}{0}$ & $\underset{r}{\circ}$ \\
\hline 0 & $\stackrel{+}{0}$ & $\frac{\sqrt{2}}{\stackrel{\delta}{\nwarrow}}$ & ई̊) & $\begin{array}{l}\mathbb{0} \\
0\end{array}$ & 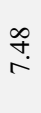 & ஸे? & $\stackrel{\circ}{n}$ & $\stackrel{n}{0}$ & $\sqrt[n]{r}$ & $\stackrel{m}{?}$ & $\stackrel{\circ}{\sim}$ & $\stackrel{n}{\tilde{n}}$ & $\underset{\sim}{\sigma}$ & ֻุ & 守 & $\stackrel{n}{0}$ & $\underset{r}{\sigma}$ & $\stackrel{n}{\dddot{n}}$ & $\underset{\sim}{\sigma}$ & $\stackrel{\overbrace{}}{0}$ & $\underset{r}{\text { గె }}$ \\
\hline$\Xi$ & $\stackrel{+}{0}$ & $\frac{\hat{n}}{\stackrel{2}{0}}$ & ஜే & $\stackrel{1}{0}$ & Oे & $\stackrel{\nabla}{\nabla}$ & $\underset{r}{\mathbb{r}}$ & $\stackrel{n}{n}$ & $\underset{⿱ 亠}{\stackrel{\infty}{\sim}}$ & ़ֻ & $\stackrel{n}{n}$ & 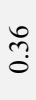 & హิ & $\stackrel{\text { กै }}{0}$ & $\begin{array}{l}\bar{\sigma} \\
\end{array}$ & $\stackrel{m}{0}$ & ñ & 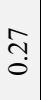 & $\stackrel{n}{n}$ & 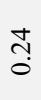 & $\stackrel{\bar{n}}{r}$ \\
\hline$\infty$ & $\stackrel{\nabla}{\circ}$ & $\frac{n}{\frac{n}{m}}$ & ई̊) & $\begin{array}{l}\stackrel{8}{\circ} \\
\stackrel{0}{0}\end{array}$ & $\begin{array}{l}\sigma \\
r\end{array}$ & $\stackrel{+}{+}$ & $\stackrel{\Re}{\stackrel{2}{r}}$ & $\stackrel{+}{\oplus}$ & $\underset{r}{\mathbb{N}}$ & $\begin{array}{l}0 \\
\text { ?ִ } \\
0\end{array}$ & $\stackrel{\substack{n \\
r}}{ }$ & $\stackrel{m}{\dddot{m}}$ & $\underset{\sigma}{\sigma}$ & @़ & $\stackrel{n}{n}$ & $\stackrel{\infty}{\stackrel{\infty}{0}}$ & $\stackrel{n}{n}$ & $\stackrel{\overbrace{}}{0}$ & $\begin{array}{r}\sigma \\
\sim\end{array}$ & $\underset{\sim}{\widetilde{N}}$ & $\stackrel{\infty}{\mathfrak{n}}$ \\
\hline 2 & $\stackrel{\nabla}{0}$ & 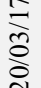 & ई્ळ & $\stackrel{a}{\circ}$ & $\hat{n}$ & $\stackrel{\circ}{+}$ & $\stackrel{\circ}{n}$ & 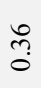 & $\begin{array}{l}\infty \\
\sim \\
r\end{array}$ & m? & $\underset{r}{r}$ & $\stackrel{m}{0}$ & $\stackrel{n}{n}$ & $\stackrel{\infty}{\stackrel{\infty}{0}}$ & $\underset{r}{\stackrel{g}{r}}$ & $\stackrel{\text { ஸे }}{0}$ & 广) & $\underset{0}{\widetilde{o}}$ & 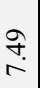 & 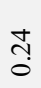 & $\begin{array}{l}\stackrel{0}{n} \\
r\end{array}$ \\
\hline 가 & $\stackrel{+}{0}$ & 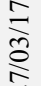 & 용 & $\begin{array}{l}\stackrel{8}{0} \\
\stackrel{0}{0}\end{array}$ & n़े & సે? & $\underset{r}{\infty}$ & $\stackrel{+}{3}$ & $\underset{r}{\sigma}$ & m? & in & m? & $\stackrel{n}{n}$ & กิ & 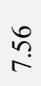 & $\stackrel{2}{n}$ & in & $\frac{9}{0}$ & $\stackrel{\infty}{n} \underset{n}{n}$ & $\stackrel{\widetilde{N}}{0}$ & $\stackrel{n}{n}$ \\
\hline $\bar{\sim}$ & $\stackrel{+}{0}$ & 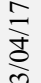 & ई̊) & $\stackrel{\infty}{\circ}$ & $\underset{r}{n}$ & $\stackrel{8}{8}$ & $\stackrel{+}{r}$ & $\stackrel{n}{n}$ & n़े & ?ै? & $\begin{array}{l}\infty \\
\stackrel{\sim}{r}\end{array}$ & กै? & $\begin{array}{l}\infty \\
n \\
\sim\end{array}$ & ?ֶ? & $\underset{\sim}{\sigma}$ & ก̊? & $\underset{\sim}{\sigma}$ & ָָ & $\stackrel{\sim}{n}$ & ֶָ & $\underset{\sim}{\sigma}$ \\
\hline$\widetilde{Z}$ & $\stackrel{+}{0}$ & $\frac{\sqrt{f}}{\stackrel{f}{\delta}}$ & ई̊ & $\stackrel{0}{0}$ & $\underset{\sigma}{\sigma}$ & $\underset{0}{\nabla}$ & $n$ & 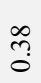 & $\underset{r}{\sigma}$ & ?ֶ? & 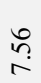 & m? & $\underset{\sim}{\sigma}$ & $\stackrel{m}{\dddot{m}}$ & $\begin{array}{l}\stackrel{n}{r} \\
\stackrel{n}{n}\end{array}$ & ?n & $\stackrel{\circ}{n}$ & సి & $\underset{\sim}{\stackrel{r}{r}}$ & స̊ & $\underset{\sim}{\stackrel{8}{\circ}}$ \\
\hline$\approx$ & $\stackrel{+}{0}$ & 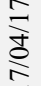 & ભే & $\stackrel{\infty}{\circ} \stackrel{0}{0}$ & $\sqrt{n}$ & $\stackrel{\infty}{m}$ & $\underset{\sim}{\sigma}$ & $\stackrel{+}{\text { m? }}$ & $\underset{\sim}{\sigma}$ & ঙ̊? & $\stackrel{n}{n}$ & กै & $\stackrel{\sim}{n}$ & ণิ & $i n$ & กొ & $\begin{array}{l}\infty \\
n \\
n\end{array}$ & 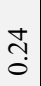 & $\underset{\sim}{\sigma}$ & ָָ & $\begin{array}{l}\mathscr{n} \\
\end{array}$ \\
\hline
\end{tabular}

The results showed that after installing the chlorine system at $0.4 \mathrm{mg} / \mathrm{l}$ chlorine concentrate, the residual chlorine concentration at positions after inlet at point 1 (just take samples at 2, 3, 4, 5, 6, 7, 8 and 9 points ) ranged from 0.3 to $0.4 \mathrm{mg} / 1, \mathrm{pH}$ in the range 7.4 $-7.8 \mathrm{pH}$. This level of chlorine and $\mathrm{pH}$ meet the standard. 


\section{Discussions}

When choosing chlorination system station location, it is necessary to consider both technical and landscape efficiency. The location of the chlorination system station should be selected in public land or infrastructure land so as avoid obstructing traffic and affect people's life, this location should be at inlet of the water supply network area or DMA (District Metering Area) so that the service area is the largest. They are should be combined with flow water meter chamber to flow signal.

In large cities as Ho Chi Minh City with a large and complex water distribution network, limit about online water quality monitoring system. It is very difficult to locate area or position that need add chlorine, the author suggests combining using Epanet software to monitor the decrease in chlorine concentration in water combined with manual water sampling to know the available chlorine concentration and calculate the chlorine concentration to increase. Moreover, water sampling should be carried out both at the inletand critical point of water supply area to ensure that chlorine is not too high at inlet and is inadequate at critical point.

The chlorination system must be regularly monitored after installation to increase or decrease chlorine concentration timely depending on water quality, because water quality may vary from season to year.

The Chlorun system is not only suitable for water supply networks and can also be used in small rural water supply stations and requires a simple and safe chlorination system solution, in water tanks in buildings and public construction where verchlorine concentrate level lower that standard requirement.

\section{Conclusions}

Besides $\mathrm{pH}$ and turbidity, residual chlorine concentration is an important factor to assess drinking water quality. Lack of residual chlorine in water is a common problem in water supply areas that are far from water supply facilities, or in long-term water storage such as cisterns. This study provides a simple solution to this problem, by installing chlorinationin granular form station at inlet of water supply network. The solution is not only applicable in Ho Chi Minh City but also can be applied in all provinces where chlorine concentrate levelsin drinking water do not meet the Ministry of Health standards.

In subsequent studies, the author will monitor decrease in chlorine concentrations in water network combined with installation of a continuous chlorine online monitoring system to determine proper the chlorination station location. Bring to the highest results in both technical and economic efficiency.

\section{References}

1. A.O. Al-Jasser, Water research 41.2, 387-396 (2007)

2. M.M. Amin, International Journal of Environmental Health Engineering 2.1, 22 (2013)

3. J.C. Block, Biofouling 6.4, 333-343 (1993)

4. G.J. Bowden, Mathematical and computer modelling 44.5, 469-484 (2006)

5. Chlorine Chemistry Council and American Chemistry Council, Drinking Water Chlorination: A Review of Disinfection Practices and Issues, Water conditioning and purification international (2006)

6. E. Delahaye, Water research 37.15, 3689-3696 (2003)

7. S. Dukan, Water Research 30.9, 1991-2002 (1996) 
8. W.E. Elshorbagy, H. Abu-Qdais, M. K. Elsheamy, Water Research 34.13, 3431-3439 (2000)

9. E.E. Geldreich, Journal American Water Works Association, 596-602 (1972)

10. D.E. Helbling, J.M. VanBriesen, Journal of Environmental Engineering 135.10, 918927 (2009)

11. M.J. Lehtola, Journal of Industrial Microbiology and Biotechnology 31.11, 489-494 (2004)

12. Li, Xin, Journal of Environmental Sciences 15.1, 136-144 (2003)

13. L. Wen, L. Kiéné, Y. Lévi, Water Research 33.3, 827-835 (1999)

14. E.D. Mintz, F.M. Reiff, R.V. Tauxe, Jama 273.12, 948-953 (1995)

15. M.J. Rodriguez, Jean-B. Sérodes, Environmental Modelling \& Software 14.1, 93-102 (1998)

16. Y.X. Sun, Journal of Hazardous Materials 168.2, 1290-1295 (2009)

17. I. Toroz, V. Uyak, Desalination 176.1-3, 127-141 (2005)

18. A. Tsintzou, Water, Air, \& Soil Pollution 120.3, 273-282 (2000)

19. C.W. White, J.G. Martin, Proceedings of the American Thoracic Society 7.4, 257-263 (2010)

20. G.R. Zhang, Environmental Technology 13.10, 937-946 (1992) 